\title{
Effetto delle dimensioni del cordone di saldatura sulla resistenza a fatica dei giunti a croce
}

\author{
B. Atzori, B. Rossi \\ Università di Padova, Dipartimento di Ingegneria Meccanica, bruno.atzori@unipd.it \\ G. Demelio \\ Politecnico di Bari, Dipartimento di Ingegneria Meccanica e Gestionale, demelio@poliba.it
}

RiASsUNTO. La scelta della dimensione da assegnare al cordone di saldatura nel caso di giunzioni saldate a croce può presentarsi problematica, specie quando gli spessori delle lamiere che formano il giunto sono differenti tra loro. Le normative di tipo tecnologico suggeriscono in genere di prevedere uno spessore del cordone di saldatura inferiore al minimo spessore della lamiera da collegare, mentre le normative di tipo strutturale non prevedono una dipendenza della resistenza del giunto, sia statica che a fatica, dalle dimensioni del cordone di saldatura, a meno che queste non siano tanto ridotte da portare a rotture che si inneschino e si propaghino non più nella lamiera base ma nel cordone di saldatura. Scopo del presente lavoro è la verifica, teorica e sperimentale, della variazione della resistenza a fatica al variare del rapporto tra lo spessore del cordone di saldatura e quello minimo delle lamiere da saldare.

ABSTRACT. The choice of the proper weld size for cruciform joints can be a critical topic especially in case of different thickness of the welded plates. According to technological recommendations the size of the weld bead should not exceed the thickness of the smallest plate. On the other hand, design standards do not suppose the joint static and fatigue resistance to be dependent on the weld size provided that it is thick enough to avoid the failure of the weld itself instead of the failure of the welded plate. The aim of this work is to study, both from a theoretical and an experimental point of view, the effect of different weld sizes on the fatigue resistance of cruciform joints.

Parole Chiave. Fatica; Giunti saldati a croce; Dimensioni del cordone; Fattore di intensificazione delle tensioni

\section{INTRODUZIONE}

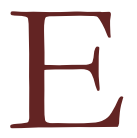

' ben noto che, nel caso di giunti a croce portanti saldati con filetti d'angolo, dovrebbe essere sufficiente uno spessore del cordone di saldatura pari alla metà dello spessore della lamiera a cui è applicato il carico per ristabilire la resistenza della lamiera stessa. Nella pratica applicativa non è però infrequente il caso di cordoni di saldatura di dimensioni maggiori, sia perchè espressamente previsti, per evitare il rischio di rotture per fatica che si inneschino alla radice del cordone di saldatura e si propaghino attraverso il cordone di saldatura stesso (molto più insidiose di quelle che si innescano al piede del cordone di saldatura e si propagano nel materiale base), sia anche non previsti, ma conseguenza delle modalità di saldatura adottate. Le normative per la progettazione delle strutture in acciaio (in particolare la CNR-UNI 10011 [1], la DIN 15018 [2], nonché le Raccomandazioni dell'International Institute of Welding [3]) non fanno dipendere la previsione della vita a fatica di un giunto saldato dalle dimensioni del cordone di saldatura, tranne nel caso in cui questo non sia tanto ridotto da portare ad una maggiore criticità della radice del cordone di saldatura rispetto a quella del piede, con conseguente possibile cedimento del cordone e non della lamiera base. 
Scopo del presente lavoro è l'analisi, sia teorica che sperimentale, dell'influenza dello spessore del cordone di saldatura sulla resistenza a fatica dei giunti saldati a croce, onde chiarire se il non tenerne conto, da parte delle normative di progettazione, sia giustificato o rappresenti invece un errore da correggere.

\section{ANALISI TEORICA}

L e normali tecnologie di saldatura portano alla formazione di un cordone di saldatura che si raccorda con la lamiera base in maniera brusca, con raggi di raccordo di entità molto limitata (dell'ordine dei decimi di millimetro) ai quali corrisponde un effetto di intaglio molto elevato, al contrario di quanto avviene con tecnologie o procedimenti particolari, atti ad aumentare questo raggio di raccordo, rendendolo più dolce (dell'ordine di alcuni millimetri) e riducendo così l'effetto di intaglio, con conseguente aumento della resistenza a fatica.

E' stato evidenziato da tempo come, solo nel caso delle tecnologie di saldatura normali, la resistenza a fatica sia determinata dal campo di tensione che si ha in prossimità del piede del cordone di saldatura (poco dipendente dal valore del raggio di raccordo, purché piccolo), e non dal picco valutato in campo lineare elastico, molto dipendente invece dal raggio di raccordo $[4,5]$. Tale campo di tensione presenta un andamento esponenziale, analogo a quello che si ha in prossimità di una cricca nel caso della meccanica della frattura, ma con un esponente diverso, non più pari a 0.5 ma dipendente dall'ampiezza dell'angolo formato dal cordone di saldatura con la lamiera base. Nel caso di un cordone simmetrico rispetto alle due lamiere disposte ortogonali, con un'inclinazione di $45^{\circ}$ rispetto a ciascuna lamiera e quindi con angoli di apertura pari a $135^{\circ}$, l'esponente risulta pari a 0.326 , in analogia a quanto trovato da Williams [6] per gli intagli acuti con uguale angolo di apertura.

L'approccio che studia le capacità di resistenza a fatica delle strutture saldate basandosi su questi concetti, denominato NSIF (Notch Stress Intensity Factor), è stato sviluppato e formalizzato da diversi autori [7-19]. In particolare, Lazzarin e Tovo [12] lo hanno applicato all'analisi teorica dei giunti saldati a croce, giungendo ad esplicitare l'intensità del campo di tensione locale in funzione della forma del giunto, cioè, con riferimento alla Fig. 1, ai rapporti T/t tra gli spessori delle lamiere e b/t tra il piede del cordone di saldatura e lo spessore t della lamiera soggetta al carico.

Senza entrare in questa sede in maggiori dettagli sulla trattazione effettuata da Lazzarin e Tovo, che esulerebbe dagli obiettivi della presente analisi, i risultati da loro raggiunti possono essere applicati al caso in esame, evidenziando la variazione relativa del campo di tensione (e quindi della resistenza a fatica ad un determinato numero di cicli) al variare dei rapporti dimensionali sopra ricordati. Inoltre, poiché al variare della criticità del giunto saldato, la curva di Woehler varia semplicemente traslando parallelamente a se stessa in un diagramma doppio logaritmico [5], la variazione della resistenza a fatica è la stessa indipendentemente dalla vita prevista, purché nel campo delle vite a termine. E' pertanto possibile costruire il diagramma di Fig. 2 che, nell'ipotesi di cordone portante a piena penetrazione con innesco al piede del cordone di saldatura e propagazione nella lamiera base e pendenza del cordone stesso pari a $45^{\circ}$, riporta, per un rapporto $\mathrm{T} / \mathrm{t}$ pari a 3, la variazione della prevedibile resistenza a fatica al variare di b/t. La resistenza a fatica è espressa in termini di range di sollecitazione $\Delta \sigma$, pari alla differenza tra la $\sigma$ massima e la $\sigma$ minima del ciclo di sollecitazione, supposto costante nel tempo, ed è riferita al valore $\Delta \sigma_{1}$ assunto nel caso di b $=\mathrm{t}$. L'analisi teorica, che è in corso di approfondimento per estenderla al caso di saldatura a parziale penetrazione e a valori più elevati del rapporto b/t, sembra indicare un leggero miglioramento della resistenza a fatica al crescere dello spessore del cordone di saldatura.

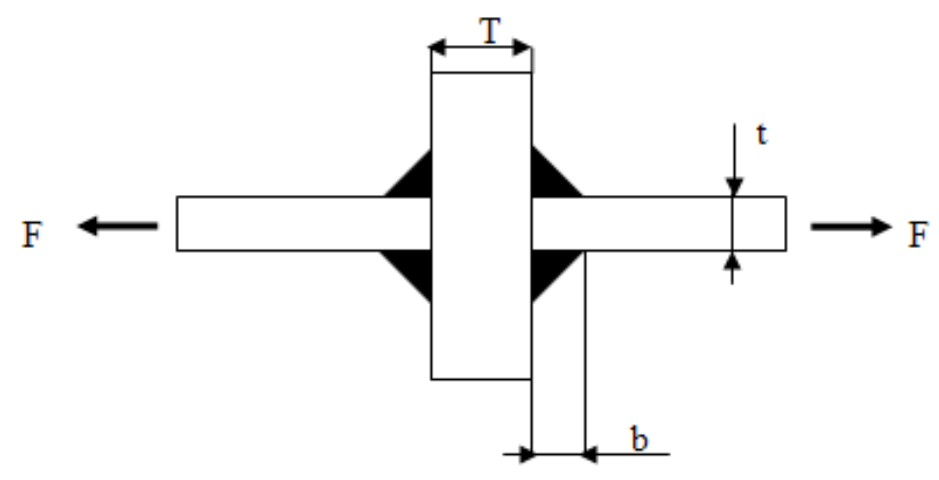

Figura 1: Tipologia di giunto saldato analizzato. 


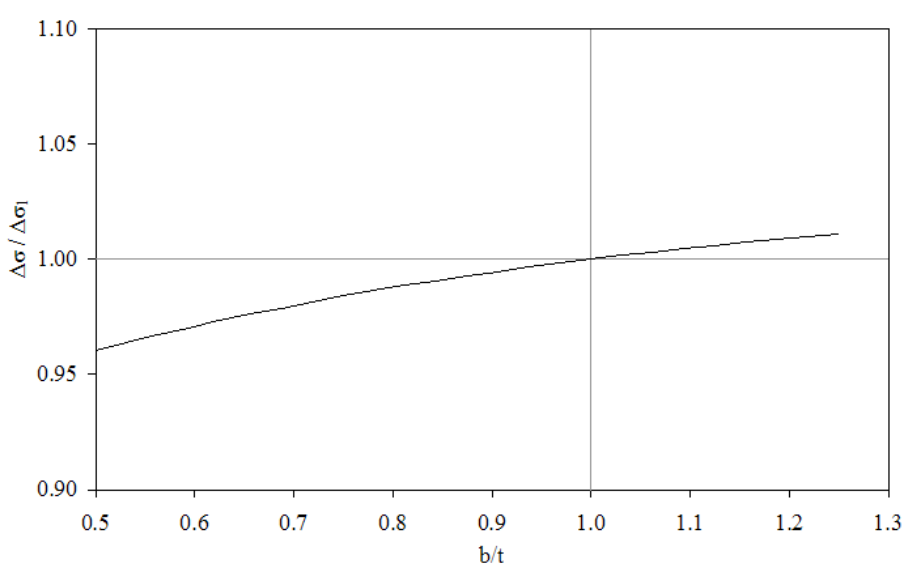

Figura 2: Variazione prevedibile della resistenza a fatica $\Delta \sigma$ a parità di numero di cicli $\mathrm{n}$ al variare dello spessore b del cordone di saldatura, per $\mathrm{T} / \mathrm{t}=3$. Valore di riferimento $\Delta \sigma_{1}$ relativo al caso $\mathrm{b} / \mathrm{t}=1$. Saldatura a completa penetrazione.

\section{Prove SPERIMENTALI}

$\mathrm{L}$

e prove sperimentali sono state effettuate su giunti saldati a croce in acciaio da carpenteria Fe 510. I giunti sono stati ottenuti saldando con cordoni d'angolo due lamiere principali, di spessore pari a $3 \mathrm{~mm}$, su una lamiera trasversale, di spessore pari a $10 \mathrm{~mm}$. Sono state realizzate due tipologie di cordoni, variandone lo spessore. Il piede del cordone di saldatura, indicato in Fig. 3 ed utilizzato per quantificare la dimensione del cordone stesso, ha una dimensione media di $4 \mathrm{~mm}$ nella prima serie e di $7 \mathrm{~mm}$ nella seconda serie, mentre l'inclinazione dei cordoni non si discosta molto dai $45^{\circ}$ per entrambe le serie. La larghezza nominale del giunto è pari a $50 \mathrm{~mm}$ ed i raggi di raccordo al piede del cordone di saldatura sono di entità molto ridotta, come evidente anche dalla Fig. 3, non essendo stato effettuato alcun trattamento per renderli più ampi.

Prima di effettuare le prove di fatica, tre provini sono stati sottoposti a trazione statica ed hanno manifestato un comportamento regolare, con rottura della lamiera principale da $3 \mathrm{~mm}$, a cui è stato applicato il carico, e non dalla saldatura. I risultati delle prove statiche sono riportati nella Fig. 4.

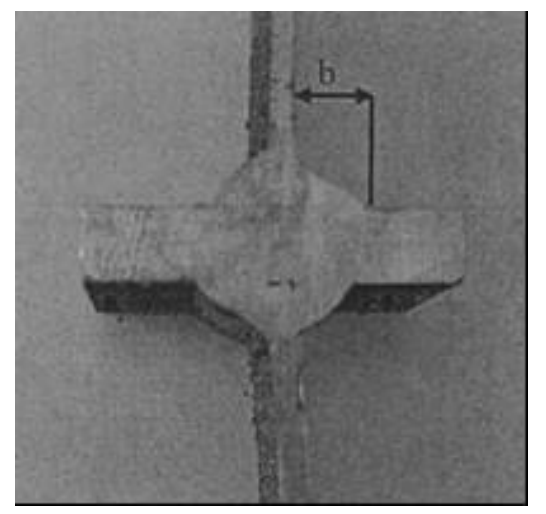

Figura 3: Geometria dei provini - sezione trasversale.

Le prove di fatica sono state effettuate utilizzando una macchina servo-idraulica Instron 1342 controllata da elettronica MTS. Tutte le prove sono state condotte in controllo di carico, con frequenze variabili tra 8 e $18 \mathrm{~Hz}$ e con rapporto di sollecitazione $\mathrm{R}=\sigma_{\min } / \sigma_{\max }=0.1$. In Tab. 1 sono riportati $\mathrm{i}$ valori dell'ampiezza di sollecitazione e del numero di cicli che hanno portato a rottura i singoli provini.

I risultati sono stati riportati in Fig. 5 e a ciascuna serie è stata applicata la curva di Woehler standard al 50\% di probabilità di sopravvivenza determinata recentemente da Atzori e Meneghetti [20]. Risulta evidente una certa differenza tra le due 
serie di risultati, che conferma le indicazioni fornite dall'analisi teorica: la resistenza a fatica migliora al crescere dello spessore del cordone di saldatura.
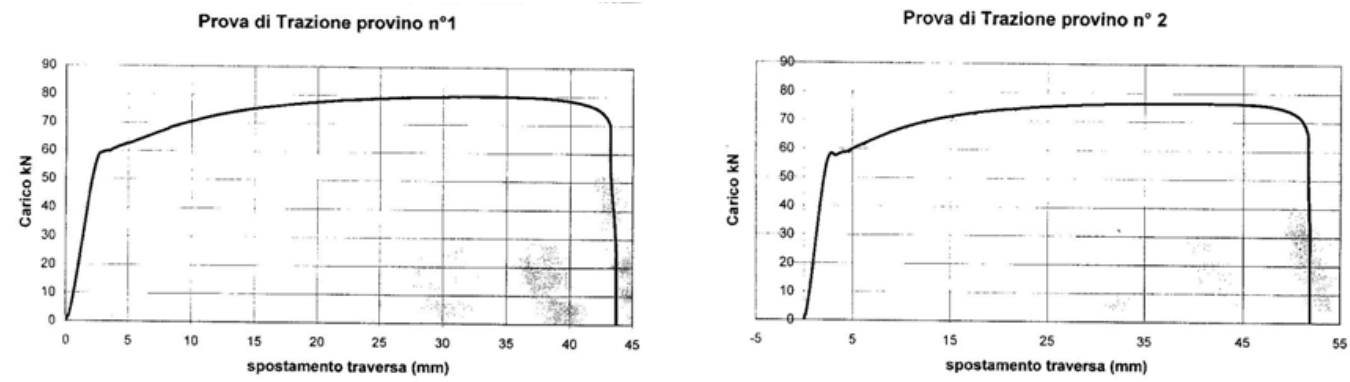

Prova di Trazione provino $n^{\circ} 3$

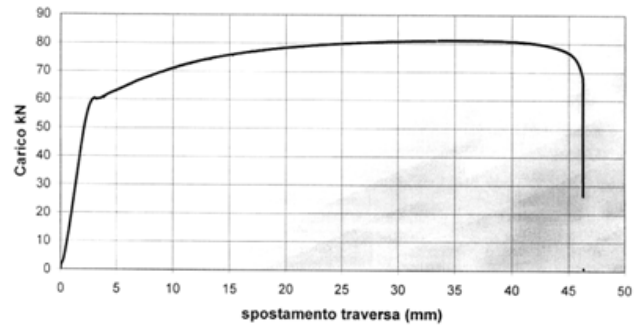

Figura 4: Risultati delle prove di trazione statica.

\begin{tabular}{lll|lll}
\hline \multicolumn{2}{l|}{ Serie $1(\mathrm{~b}=4 \mathrm{~mm})$} & & \multicolumn{3}{|l}{ Serie $2(\mathrm{~b}=7 \mathrm{~mm})$} \\
Provino & $\Delta \sigma[\mathrm{MPa}]$ & $\mathrm{N}$ & Provino & $\Delta \sigma[\mathrm{MPa}]$ & $\mathrm{N}$ \\
1 & 270 & 40700 & 1 & 297 & 36590 \\
2 & 270 & 45700 & 2 & 297 & 85000 \\
3 & 225 & 70900 & 3 & 297 & 45000 \\
4 & 225 & 37800 & 4 & 297 & 43945 \\
5 & 180 & 149900 & 5 & 225 & 72000 \\
& & & 6 & 225 & 131000 \\
& & & 7 & 180 & 163000 \\
\hline
\end{tabular}

Tabella 1: Risultati delle prove di fatica.

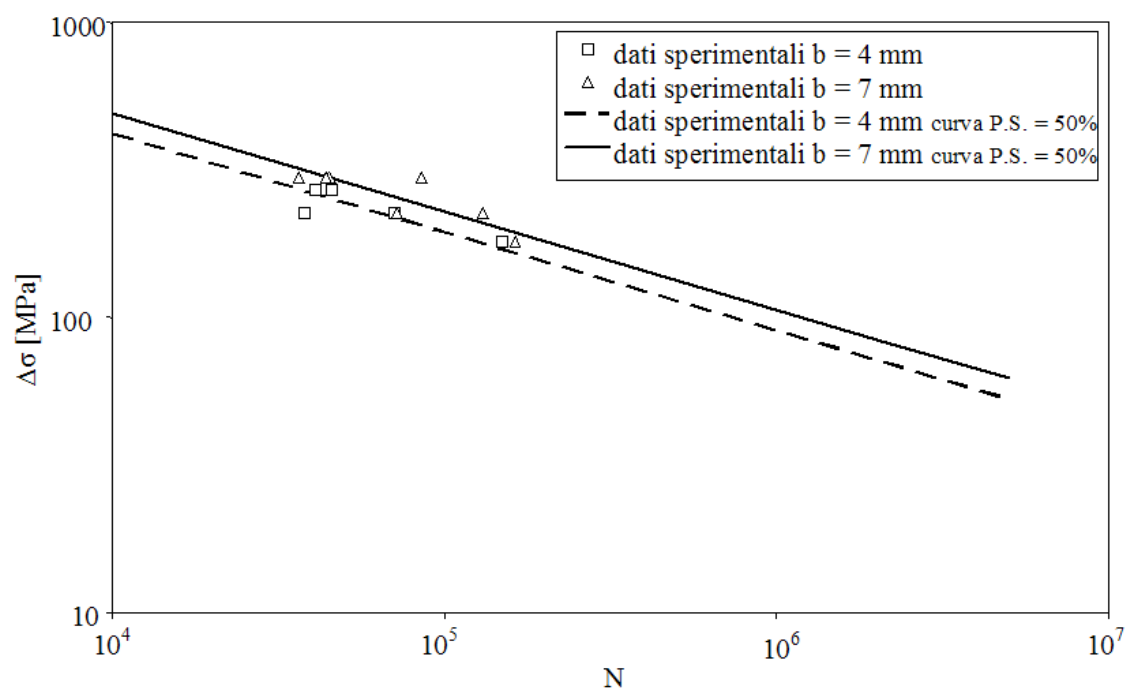

Figura 5: Risultati delle prove di fatica. 
Poiché però la differenza di comportamento è modesta ed il numero di dati sperimentali per ora disponibili è limitato, i risultati delle due serie sono stati considerati come appartenenti ad un'unica popolazione e ad essi è stata applicata la banda di dispersione statistica recentemente determinata da Atzori e Meneghetti [20] e riportata in Fig. 6. In Fig. 7 è mostrato il risultato ottenuto.

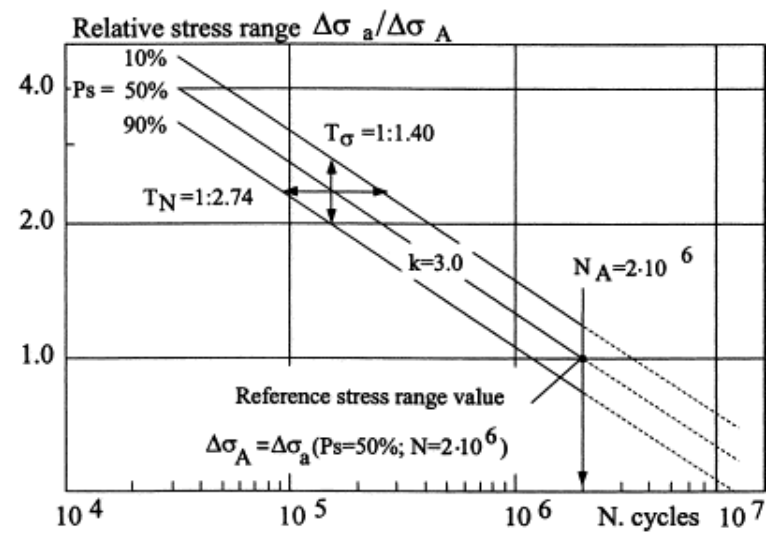

Figura 6: Banda di dispersione unificata per giunti in acciaio con cordoni d'angolo [20].

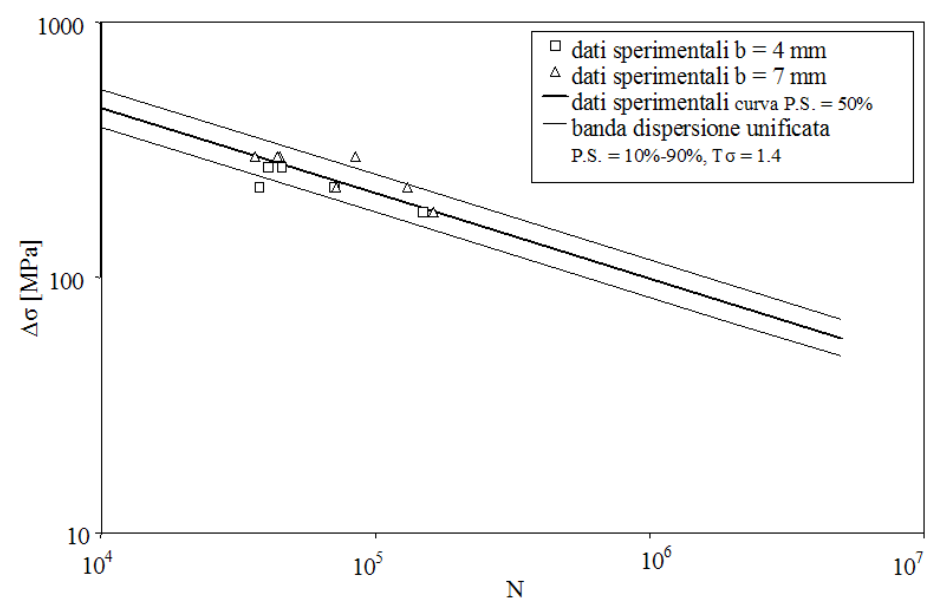

Figura 7: Banda di dispersione unificata applicata ai risultati sperimentali.

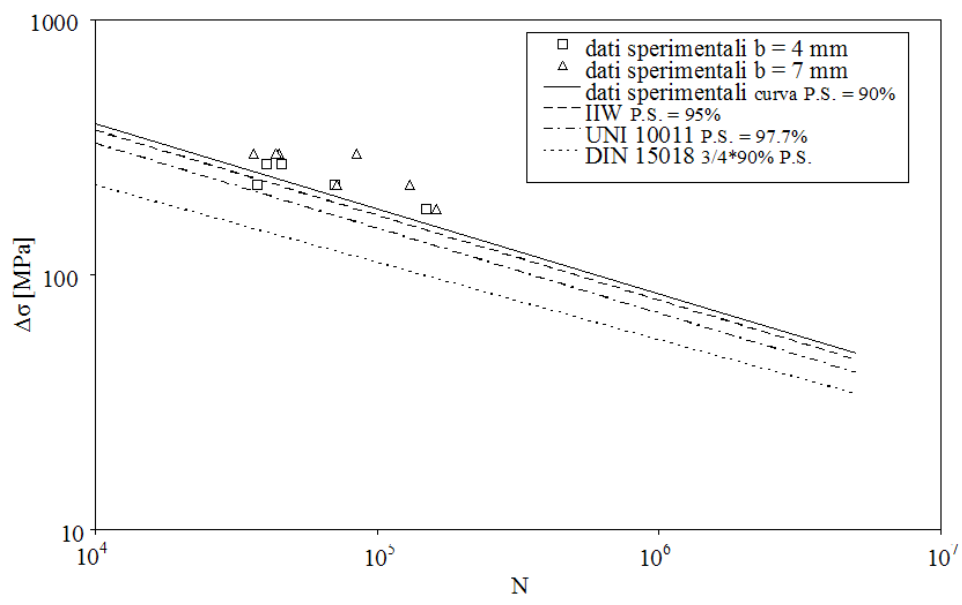

Figura 8: Confronto tra normative e risultati sperimentali. 
Poiché la banda di dispersione ha un limite inferiore relativo ad una probabilità di sopravvivenza (P.S.) del 90\%, la curva di resistenza a fatica ricavata dai risultati sperimentali e relativa a tale probabilità di sopravvivenza è stata confrontata con le curve di progetto fornite, per giunti della stessa tipologia, da alcune normative. In particolare si riportano in Fig. 8 i valori relativi a P.S. $=90 \%$ moltiplicati per $3 / 4$ secondo l'approccio di determinazione dei valori di resistenza della DIN 15018, quelli relativi a P.S. $=95 \%$ secondo l'approccio IIW e quelli relativi a P.S. $=97.7 \%$ secondo l'approccio CNR-UNI 10011.

\section{CONCLUSIONI}

$\mathrm{S}$ ia le analisi teoriche che i risultati sperimentali ottenuti mostrano che all'aumentare dello spessore del cordone di saldatura la resistenza a fatica del giunto saldato non diminuisce ma anzi migliora.

Poiché il miglioramento, almeno per ora, sembra essere modesto, appare giustificato ed ammissibile che le normative non tengano conto delle dimensioni del cordone di saldatura nel fissare la resistenza a fatica dei giunti saldati con cordoni d'angolo.

I risultati sperimentali ottenuti evidenziano caratteristiche di resistenza congruenti ed entro i limiti di resistenza indicati, per la stessa tipologia di giunto, dalle normative di progettazione e verifica considerate.

L'analisi svolta evidenzia una non banale differenza della resistenza di progetto a fatica fornita dalle tre fonti prese in esame. Tali differenze non sembrano giustificabili con le modeste differenze delle probabilità di sopravvivenza a cui fanno riferimento le tre normative e, a parere degli autori di questo lavoro, merita un maggiore approfondimento che estenda il confronto anche ad altre normative, visto il rischio da un lato, di avere un inutile appesantimento delle strutture (con conseguente maggior costo e minore competitività sul mercato), dall'altro, di avere rotture catastrofiche dopo periodi anche lunghi di utilizzo delle strutture.

\section{BIBLIOGRAFIA}

[1] CNR UNI 10011 Costruzioni di acciaio. Istruzioni per il calcolo, l'esecuzione, il collaudo e la manutenzione (1988).

[2] DIN 15018 Steel structures Verification and analyses. Part 1 (1984).

[3] A. Hobbacher Fatigue design of welded joints and components. Recommendations of IIW Joint Working Group XIIIXV (1996).

[4] B. Atzori, G. Crivellato, G. Meneghetti, Atti del XXVI Convegno Nazionale AIAS, Calabria (1997).

[5] B. Atzori, Rivista Italiana della Saldatura LII, 1 (2000) 27.

[6] ML. Williams, J. Appl. Mech. 19 (1952) 526.

[7] E. Haibach, Die Schwingfestigkeit von Schweissverbindungen aus der Sicht einer örtlichen Beanspruchungsmessung. LBF Report No. FB-77, Darmstadt (1968).

[8] S. Usami, H. Kimoto, S. Kusumoto, Trans Jpn Weld Soc., 9(2) (1978).

[9] B. Atzori, G. Blasi, C. Pappalettere, Exp. Mech., 25(2) (1985) 129.

[10] L.S. Nui, C. Chehimi, G. Pluvinage, Engng Fracture Mech., 49 (1994) 325.

[11] Y. Verreman, B. Nie, Fatigue Fract. Engng Mater. Struct., 19 (1996) 669.

[12] P. Lazzarin, R. Tovo, Fatigue Fract. Engng. Mater. Struct. 21 (1998) 1089.

[13] B. Atzori, P. Lazzarin, G. Meneghetti, Fatigue Fract. Eng. Mater. Struct., 22 (1999) 369.

[14] R. Tovo, P. Lazzarin, Int. J. Fatigue, 21 (1999) 1063-78.

[15] P. Lazzarin, P. Livieri, Int. J. Fatigue, 23(3) (2001) 225-32.

[16] G. Meneghetti, R. Tovo, in: Blom AF, editor. Proceedings of the $8^{\text {th }}$ International Fatigue Congress. Stockholm, Sweden. (2002)1873.

[17] D. Taylor, N. Barrett, G. Lucano, Int. J. Fatigue, 24 (2002) 509-18.

[18] Y. Verreman, N. Limodin, Eng. Fract. Mech., (2007) doi:10.1016/j.engfractmech.2007.07.005.

[19] B. Atzori, P. Lazzarin, G. Meneghetti, Eng Fract Mech., (2007) doi:10.1016/j.engfracmech.2007.03.029.

[20] B. Atzori, G. Meneghetti, Int. J. Fatigue, 23 (2001) 713-21. 